\title{
Population Structure of the Blueberry Pathogen Monilinia vaccinii-corymbosi in the United States
}

\author{
Kathleen M. Burchhardt and Marc A. Cubeta
}

Department of Plant Pathology, North Carolina State University, Campus Box 7567, Raleigh 27695-001. Accepted for publication 5 October 2014.

\begin{abstract}
Burchhardt, K. M., and Cubeta, M. A. 2015. Population structure of the blueberry pathogen Monilinia vaccinii-corymbosi in the United States. Phytopathology 105:533-541.

The fungus Monilinia vaccinii-corymbosi causes disease of blueberry (Vaccinium section Cyanococcus) shoots, flowers, and fruit. The objective of our research was to examine the population biology and genetics of M. vaccinii-corymbosi in the United States. A total of 480 samples of M. vaccinii-corymbosi were collected from 18 blueberry fields in 10 states; one field in Georgia, Massachusetts, Maine, Michigan, Mississippi, New Jersey, New York, Oregon, and Washington and nine fields in North Carolina. Analysis with 10 microsatellite markers revealed 247 unique multilocus haplotypes (MLHs), with 244 MLHs detected within 11 fields

in the Northeast, Northwest, Midwest, and Southeast and three MLHs detected within seven fields in the Southeast United States. Genetic similarity and low genetic diversity of M. vaccinii-corymbosi isolates from the seven fields in the Southeast United States suggested the presence of an expansive, self-fertile population. Tests for linkage disequilibrium within 10 fields that contained $\geq 12$ MLHs supported random mating in six fields and possible inbreeding and/or selffertilization in four fields. Analysis of molecular variance, discriminate analysis of principal components, and Bayesian cluster analysis provided evidence for population structure and restricted gene flow among fields. This research represents the first comprehensive investigation of the genetic diversity and structure of field populations of $M$. vacciniicorymbosi.
\end{abstract}

Blueberry production has rapidly expanded in the United States from an approximately \$220 million market in 2003 to over $\$ 781$ million in 2012 (U.S. Department of Agriculture, National Agricultural Statistics Service). Demand for blueberries has driven the desire for increased yield while minimizing production costs (52). The plantpathogenic fungus Monilinia vaccinii-corymbosi (Reade) Honey causes mummy berry disease of several blueberry (Vaccinium section Cyanococcus) species throughout North America $(2,3)$ and has also been reported in Europe (22). The fungus causes shoot blight followed by ovary infection through the gynoecial pathway, leading to mummification of infected fruit (i.e., pseudosclerotia or "mummies"). The three primary marketed species of blueberry, $V$. corymbosum (northern highbush) and associated hybrids (e.g., $V$. corymbosum $\times V$. darrowii, a southern highbush hybrid), $V$. virgatum (synonym V. ashei, rabbiteye), and V. angustifolium (lowbush), are susceptible to shoot and fruit infection by $M$. vaccinii-corymbosi $(2,3)$. High disease incidence can lead to substantial fruit yield loss $(2,24,40)$. While M. vaccinii-corymbosi is an economically significant pathogen on blueberry, virtually nothing is known about the population biology and genetics of this fungus.

The life cycle of $M$. vaccinii-corymbosi involves production of both asexual and sexual spores (conidia and ascospores, respectively) within a single growing season and aggressive mimicry to successfully infect the ovary of blueberry $(2,35)$. Pseudosclerotia from the previous growing season serve as overwintering structures and produce sexual structures (apothecia) and wind-disseminated ascospores that infect blueberry shoots in early spring $(2,10)$. Infected tissues become blighted and conidia are produced after a latent period of approximately 1 to 2 weeks (2). Pollinating insects are attracted to infected shoots through production of

Corresponding author: K. M. Burchhardt;

E-mail address: kathleen.burchhardt@gmail.com

http://dx.doi.org/10.1094/PHYTO-03-14-0074-R

(C) 2015 The American Phytopathological Society olfactory cues and also by mimicry as conidia reflect UV light at the same wavelength as blueberry calyxes $(4,48)$. Pollinators deposit conidia on the stigma of blueberry flowers, with the conidia then displaying aggressive mimicry to infect the ovary through the gynoecial pathway $(35,36)$. The length of time that newly opened blueberry flowers are receptive to conidia is short, with research suggesting a linear decrease in infection success over 4 days postanthesis (37). Hyphae of M. vaccinii-corymbosi colonize locules of infected fruit and cause desiccation resulting in the formation of a hardened pseudosclerotium that detaches from the bush, with continuation of the life cycle the following spring.

An important component of the disease ecology and population biology of plant-pathogenic fungi is spore dispersal capability, which contributes to gene flow among populations. Previous research has suggested that dispersal of ascospores and conidia of $M$. vaccinii-corymbosi occurs over short distances. Cox and Scherm (10) found that $95 \%$ of fruit infection occurred within $20 \mathrm{~m}$ of a conidial inoculum source, with increased prevalence of disease upwind suggesting the importance of bees as vectors since they are known to forage upwind. These researchers also found that $95 \%$ of ascospores were deposited within $30 \mathrm{~m}$ of their source. There was also a positive correlation between dispersal distance and wind speed indicating the role of wind in the dissemination of ascospores. However, a long tail in the dispersal gradient of ascospores suggested that they are capable of traveling further distances. Dispersal of ascospores and/or conidia followed by successful colonization and reproduction on the host contributes to gene flow among fields. Analysis of genetic structuring of populations of $M$. vaccinii-corymbosi can be used to deduce the extent of gene flow between and among blueberry fields.

M. vaccinii-corymbosi has evolved to synchronize its developmental biology with that of its plant host(s) $(30,31,46)$. Vaccinium spp. require a preset number of hours below a threshold temperature (i.e., chill-hours) for flower bloom (53). Vaccinium virgatum cultivars require considerably fewer chill-hours than $V$. corymbosum, restricting their production mainly to the Southeast United States, 
while $V$. corymbosum is commonly grown in the Midwest, Northeast, and Northwest United States $(46,53)$. Interestingly, studies examining timing of apothecia formation from pseudosclerotia report that pseudosclerotia have chill-hour requirements that are comparable to their plant host, with pseudosclerotia formed on $V$. virgatum requiring substantially fewer chill-hours than pseudosclerotia formed on $V$. corymbosum to produce apothecia $(30,31,46)$. Significant variation in timing of apothecia production of pseudosclerotia formed on two cultivars of $V$. corymbosum displaying differences in timing of fruit development has also been reported (30,31). The mechanism(s) associated with timing of apothecia production on different Vaccinium spp. and cultivars with different developmental phenologies is unknown, but research suggests that variation in timing of apothecia production may be at least partially under genetic control within populations of $M$. vaccinii-corymbosi (31).

The objective of this study was to conduct a comprehensive investigation of intraspecific genetic diversity, population structuring, and reproductive biology of $M$. vaccinii-corymbosi in blueberry fields throughout the United States. Given the highly specific nature of infection by $M$. vaccinii-corymbosi, variation in developmental biology within populations of $M$. vacciniicorymbosi, and evidence for limited spore dispersal, we hypothesized that populations of $M$. vaccinii-corymbosi are genetically structured. We used polymorphic microsatellite markers that we previously developed for $M$. vaccinii-corymbosi to address our objectives (34). To our knowledge, this is the first comprehensive study to report high intraspecific genetic diversity and structuring of populations of $M$. vaccinii-corymbosi.

\section{MATERIALS AND METHODS}

Sample collection, isolation of $M$. vaccinii-corymbosi, and DNA extraction. Samples of infected fruit and/or shoots were collected from 18 blueberry fields in 10 states; one field each in Georgia (GA), Massachusetts (MA), Maine (ME), Michigan (MI), Mississippi (MS), New Jersey (NJ), New York (NY), Oregon (OR), and Washington (WA) (Table 1). Blueberry fields were selected with a known history of mummy berry disease. Field sites were selected to capture the geographic range of blueberry production and the three primary blueberry species grown in the continental United States; V. corymbosum (and V. corymbosum hybrids), $V$. virgatum, and $V$. angustifolium. Sampling was conducted between February and August from 2010 to 2012. Depending on the size of the blueberry field and presence of disease within the field, sampling was conducted either over the entire field or within a region with high incidence of disease. The sampling protocol at each field was designed to systematically collect samples from several rows of planted blueberry bushes and several bushes within rows. Due to differences in size of fields, we modified the number of sampled rows, bushes sampled within each row, and interval between sampled bushes at each field location to best cover the geographic extent of the selected area. Typically five rows were selected for sampling, and within each row at least one infected fruit or shoot would be sampled from a bush near the beginning of the row. Bushes were subsequently systematically sampled in the row at an interval of at least two bushes (approximately $3 \mathrm{~m}$ ) between sampled bushes. Samples from ME were collected from a wild, unmanaged population of $V$. angustifolium along three transects, with samples collected from bushes spaced $15 \mathrm{~m}$ apart along each transect. Samples were stored in Ziploc bags, placed on ice in the field, and stored at $4^{\circ} \mathrm{C}$ until they were processed.

To isolate $M$. vaccinii-corymbosi from infected shoots, tissues with conidia were lightly tapped over a Petri dish (60-mm-diameter) containing half-strength potato dextrose agar (PDA) amended to contain $2 \%$ agar (12 $\mathrm{g}$ of Difco potato dextrose broth [PDB] and $20 \mathrm{~g}$ of Difco agar per liter [Becton, Dickinson and Company, MD]) to disperse conidia on the surface of the medium. After incubation for at least $12 \mathrm{~h}$ at room temperature (approximately $20^{\circ} \mathrm{C}$ ), an individual germinating conidium was transferred with a scalpel under a dissecting microscope to either half-strength PDA containing $1.5 \%$ agar (15 g of Difco agar per liter) or half-strength malt extract agar (16.8 $\mathrm{g}$ of Difco malt extract broth and $15 \mathrm{~g}$ of Difco agar per liter [Becton, Dickinson and Company]). If a single conidium isolate was not generated due to low germination rates or survival

TABLE 1. Locality and sampling information for the 18 fields where samples of Monilinia vaccinii-corymbosi were collected ${ }^{\mathrm{a}}$

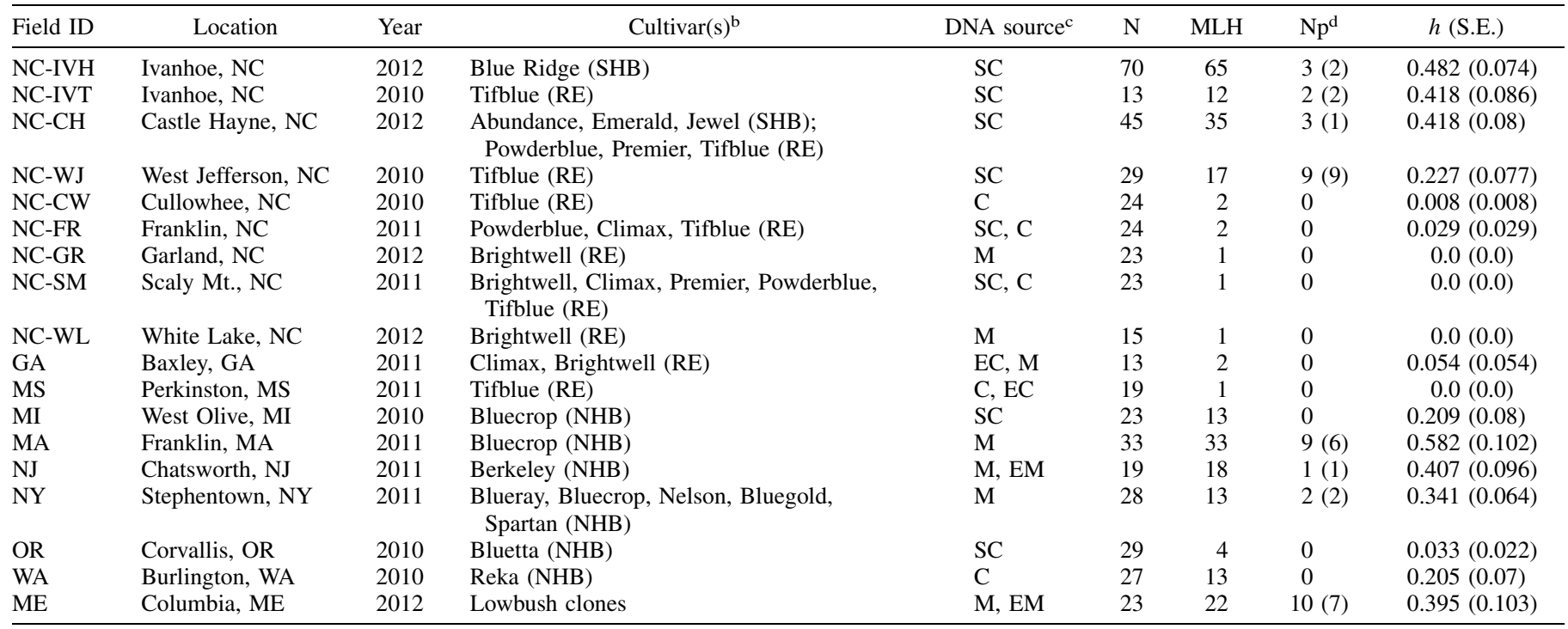

${ }^{a}$ Location of the 18 fields, year samples were collected, blueberry host cultivar, DNA source of the samples, number of samples (N), multilocus haplotypes $(\mathrm{MLH})$, private alleles $(\mathrm{Np})$, and unbiased haploid diversity $(h)$ with standard error (S.E.).

b Abbreviations for species of the cultivar(s): SHB = Vaccinium corymbosum $\times$ V. darrowii (southern highbush), NHB $=$ V. corymbosum (northern highbush), $\mathrm{RE}=$ V. virgatum (rabbiteye).

c Abbreviations for source of the DNA of the samples: $\mathrm{SC}=$ single conidium isolate, $\mathrm{C}=$ isolate derived from several conidia, $\mathrm{EC}=\mathrm{DNA}$ extracted directly from conidia, $\mathrm{M}$ = isolate cultured from mycelium inside a pseudosclerotium, EM = DNA extracted directly from mycelium inside a pseudosclerotium.

${ }^{d}$ Number in parentheses represents the number of private alleles with a frequency of $>0.05$ in the field. 
of hyphae, a colony of germinated conidia was transferred to obtain a culture. To isolate M. vaccinii-corymbosi from fruit in early stages of infection, the outside surface of an individual fruit was disinfested for $30 \mathrm{~s}$ in $10 \%$ bleach $(\mathrm{NaOCl})$ followed by $30 \mathrm{~s}$ in $70 \%$ ethanol and then rinsed in sterilized water for $30 \mathrm{~s}$. Infected fruit were cut in half with a sterile scalpel and a piece of fungal mycelium was removed from a single locule and transferred to half-strength PDA containing $1.5 \%$ agar.

All isolates were grown at room temperature for approximately 2 weeks before being used to inoculate $250-\mathrm{ml}$ Erlenmeyer flasks containing $50 \mathrm{ml}$ of half-strength PDB (12 $\mathrm{g}$ of Difco PDB per liter). After approximately 2 weeks, mycelium of each isolate was vacuum-filtered and squeeze dried. DNA was extracted from approximately $15 \mathrm{mg}$ of mycelium according to the cetyltrimethylammonium bromide (CTAB) and chloroform DNA extraction method outlined by Kretzer et al. (29), with modifications and DNA precipitation as recommended by the authors (34). Due to the inability to obtain a pure culture isolate from several samples in GA, MS, ME, and NJ, DNA was extracted directly from a small piece of tissue from a shoot with conidia or from mycelium removed from inside a single infected fruit as described above. The DNA extracts of all samples were diluted either 1:2, 1:5, or 1:10 with polymerase chain reaction (PCR) grade water and stored at $-20^{\circ} \mathrm{C}$.

PCR and fragment analysis. PCR was performed on DNA extracts using 10 microsatellite markers (Mvc7, 9, 10, 12, 15, 23, 25, 27,28 , and 30 ) developed by the authors (34). The microsatellite markers were chosen based on their ability to distinguish genetically different isolates and high levels of polymorphism. Primer sets for two or three markers were combined (i.e., multiplexed) in PCR reactions, with Mvc7, 9, and 15 (Mvc7-9-15) and Mvc10, 25, and 27 (Mvc10-25-27) amplified in triplexed PCR reactions, and Mvc12 and 23 (Mvc12-23) and Mvc28 and 30 (Mvc28-30) in duplexed reactions. All PCR reactions were setup in 0.3 -ml 96-well PCR plates in $10 \mu \mathrm{l}$ reaction volumes. PCR products were fluorescently labeled by adding a 'CAG' sequence to the 5' end of one of the primers in each marker set and including an additional ' $\mathrm{CAG}$ ' primer with either an NED or 6-FAM fluorescent tag attached to the 5 ' end to the reaction $(18,47)$. Multiplexed PCR reactions contained 1× PCR Master Mix (reaction buffer with $0.025 \mathrm{U} / \mu \mathrm{Taq}$ DNA polymerase, $2 \mathrm{mM} \mathrm{MgCl}_{2}$, and $0.2 \mathrm{mM}$ of each dNTP; Thermo Scientific), $0.05 \mu \mathrm{M}$ each 'CAG'tailed primer, $0.2 \mu \mathrm{M}$ each nontailed primer, either $0.6 \mu \mathrm{M}$ 6-FAMlabeled 'CAG' primer (triplexed reactions) or $0.4 \mu \mathrm{M}$ NED-labeled 'CAG' primer (duplexed reactions) and $1.0 \mu$ l of diluted DNA extract. Touchdown thermocycle PCR conditions and fragment analysis were performed as previously described (34) except that PCR products from reactions Mvc7-9-15 were combined with Mvc12-23, as well as products from Mvc10-25-27 combined with Mvc28-30 before submission for fragment analysis on an Applied BioSystems 3730xl DNA Analyzer (Cornell University Life Sciences Core Laboratories Center). Allele size was determined by visually scoring peaks with Peak Scanner Software version 1.0 (Applied Biosystems by Life Technologies).

Descriptive statistics, genetic diversity, and linkage disequilibrium. The length of the allele present at the 10 microsatellite loci was used to determine the multilocus haplotype (MLH) of each sample. The software GenAlEx version $6.5(38,39)$ was used to determine the number of alleles at each locus, MLH, private alleles (i.e., alleles only detected within a single field), and calculate unbiased haploid diversity within each field. The presence of private alleles with a frequency above 0.05 was evaluated since frequency of private alleles may indicate level of gene flow among fields (49). GenAlEx was also used to generate a clone-corrected data set by detecting isolates with repeated/identical MLHs within each field and retaining one representative sample per MLH in the data set. Several subsequent analyses were performed on samples collected from 10 fields containing $\geq 12$ MLHs (NC-IVH, NC-IVT, NC-CH, NC-WJ, MI, MA, ME, NJ, NY, and WA) using a clone-corrected data set since several subsequent genetic analyses can be sensitive to low sample size and departure from random mating.

The online implement of Genepop version $4.2(42,45)$ was used to test for significant linkage disequilibrium between markers within the 10 fields containing $\geq 12$ MLHs both with and without clone-corrected data, using 10,000 dememerizations, 1,000 batches, and 10,000 iterations per batch. Significant linkage disequilibrium between markers indicates that the alleles present at the loci are not randomly associated, and therefore, may be an indicator of nonrandom mating. The Benjamini-Hochberg method (5) was implemented to correct the $P$ values for multiple comparisons and test for significant linkage disequilibrium (adjusted $P<0.05$ ).

Analyses for population structure. Analysis of molecular variance (AMOVA) was performed with GenAlEx using a clonecorrected data set and the 10 fields containing $\geq 12$ MLHs to examine hierarchical partitioning of genetic variation among the fields $(38,39)$. Results were based on 10,000 data permutations and 10,000 pairwise population permutations. Linearized $\Phi_{\mathrm{PT}}$ values (analogous to $F_{S T}$ values) calculated by AMOVA were plotted against linearized geographic distances between fields to test for isolation by distance using a Mantel test in GenAlEx with 10,000 permutations $(38,39)$. A Mantel test was performed using results from the 10 fields and separately without field WA since the field was a geographical outlier.

The Bayesian analysis program STRUCTURE version 2.3.4 (41) was used to examine the $\mathrm{K}$ number of genetic clusters (i.e., populations) supported using the clone-corrected data set consisting of the 10 fields containing $\geq 12$ MLHs. The analysis probabilistically assigns individuals into genetic clusters based on allele frequencies (41). STRUCTURE was run using the admixture model that allows for mixed ancestry with location priors, allele frequencies independent, a burn-in of 20,000, Markov chain Monte Carlo (MCMC) run 500,000 times, and 20 run repeats for each value of $\mathrm{K}$ from 1 to 12 . The Evanno $\Delta \mathrm{K}$ method as performed with STRUCTURE HARVESTER web version 0.6.93 (11) was implemented to evaluate $\mathrm{K}$ based on the rate of change in the log probability over the 20 successive runs at each value of $\mathrm{K}$ (17). The $\mathrm{K}$ value at which the log likelihood approached stationary and with the highest log likelihood were also considered when evaluating K (41). Results from STRUCTURE were visualized by first permuting the estimated cluster membership coefficient matrices with CLUMPP version 1.1.2 (26) and running the output in Distruct version 1.1 (43). Mean membership probabilities as determined by CLUMPP were also used to examine population assignment. Samples were considered a member of a particular cluster if their membership probability was $Q>0.70$.

Discriminate analysis of principal components (DAPC), a multivariate analysis, was performed in the $\mathrm{R}$ package adegenet version 1.3-6 (27,28). Since DAPC does not have evolutionary assumptions built into the analysis, the clone-corrected data set for the 18 fields was used for this analysis. The K-means method was used to determine the $\mathrm{K}$ number of clusters of genetically related MLHs in the data set based on the $\mathrm{K}$ value that maximized the variation between clusters (28). Ten separate runs of K-means were performed and the mean Bayesian information criterion (BIC) value at each value of $\mathrm{K}$ from 1 to 15 was plotted. The $\mathrm{K}$ value where the BIC value either decreased by a negligible amount or increased was used to infer the K number of clusters in the data set (28). We then used DAPC to describe clusters of genetically related samples and determine membership probabilities based on the proximity of samples to the different clusters. Results from DAPC were further examined with AMOVA by testing for genetic differentiation between isolates assigned to the K clusters. Each isolate was assigned to one of $\mathrm{K}$ clusters based on the cluster they had the highest membership probability to. Genetic differentiation between isolates of $M$. vaccinii-corymbosi assigned to different clusters was calculated with AMOVA as described above. 


\section{RESULTS}

Descriptive statistics, genetic diversity, and linkage disequilibrium. Based on analysis of 480 samples with 10 microsatellite markers, 247 unique MLHs were detected (Table 1). All markers were polymorphic and number of alleles at each locus ranged from one to 11 within fields and from six to 17 among all fields. Haplotypic diversity was low in fields MS, GA, NC-CW, NC-FR, NC-SM, NC-GR, and NC-WL (see Table 1 for abbreviated naming of fields), with a maximum of two MLHs detected within each field and a total of three MLHs (herein referred to as MLH1, MLH2, and MLH3) detected among the seven fields. MLH1 was detected within fields NC-CW, NC-GR, NC-WL, GA, and MS; MLH2 within NC-CW, NC-FR, NC-SM, and GA; and MLH3 exclusively within NC-FR. Haploid diversity within these seven fields ranged from 0 to 0.054 . Genetic diversity was also low in field OR, with only four MLHs detected and a haploid diversity of 0.033. In contrast, each of the other 10 fields (NC-IVH, NC-IVT, NC-CH, NC-WJ, MA, ME, NJ, NY, MI, and WA) had higher genetic diversity, with $\geq 12$ MLHs detected per site, haploid diversity ranging from 0.205 to 0.582 , and each MLH being unique to its field of origin except for two MLHs in WA that were also detected in OR. Private alleles were detected from samples within fields NC-IVH, NC-CH, NC-IVT, NC-WJ, MA, ME, NJ, and NY, with each of the fields containing at least one private allele with a frequency above 0.05 . When considering the seven fields in the Southeast with three total MLHs as a single population, four private alleles were detected in the population with frequencies above 0.05 . Analysis of linkage disequilibrium within the 10 fields with $\geq 12$ MLHs using a non-clone-corrected data set supported that one marker pair was in significant linkage disequilibrium within NCCH (Mvc23-25), WA (Mvc9-12), and NC-IVH (Mvc9-23), and 14 marker pairs within NY (Mvc10-12, 10-23, 12-23, 7-25, 9-25, $10-25,12-25,23-25,23-28,9-30,10-30,12-30,23-30$, and 25-30) at a Benjamini-Hochberg adjusted $P<0.05$. However, all marker pairs were in linkage equilibrium within the 10 fields when analyzing a clone-corrected data set (adjusted $P<0.05$ ).

Population structure and lack of isolation by distance. Based on AMOVA using a clone-corrected data set consisting of the 10 fields containing $\geq 12$ MLHs (NC-IVH, NC-IVT, NC-CH, NC-WJ, MA, ME, NJ, NY, MI, and WA), $57 \%$ of variance was detected within fields, while $43 \%$ was detected among fields. All pairwise field comparisons were statistically significant $(P<$ $0.0001)$ for genetic differentiation, with $\Phi_{\mathrm{PT}}$ ranging from 0.167 to 0.73 (Table 2). The Mantel tests for isolation by distance were not significant when analyzing the 10 fields $\left(R^{2}=0.0424, P=0.168\right)$ and when field WA was excluded from the analysis $\left(R^{2}=0.0706\right.$, $P=0.103$ ), indicating that geographic distance between fields was not significantly correlated with genetic distance.

Population assignment based on analysis with STRUCTURE varied among the 20 runs at each value of $\mathrm{K}$. When examining runs separately at a given value of $\mathrm{K}$, all samples collected from the same field were typically placed in the same cluster. However, their grouping into the same cluster as samples from another field sometimes varied from run to run. Also, little to no admixture was detected within fields. The Evanno $\Delta \mathrm{K}$ method, a widely applied method to determine the number of populations in a given data set, supported $\mathrm{K}=3$ populations (Fig. 1A). However, this method was not able to accurately determine $\mathrm{K}$, as two populations that were strongly genetically differentiated based on AMOVA and DAPC (see below), NC-WJ and ME, were placed in the same population. Therefore, we further explored $\mathrm{K}$ by first plotting the mean $\ln \mathrm{P}(\mathrm{K})$ at each value of $\mathrm{K}=1$ to 12 (Fig. $1 \mathrm{~B}$ ). The $\ln \mathrm{P}(\mathrm{K})$ reached approximately stationary at $\mathrm{K}=6$, with the highest mean $\ln \mathrm{P}(\mathrm{K})$ at $\mathrm{K}=9$. Similar to the approach of Rosenberg et al. (44), population assignment histograms and membership probabilities at each value of $\mathrm{K}$ from 3 to 12 were then examined for patterns in population structuring (Fig. 2). Due to lack of convergence in population assignment over multiple runs at each value of $\mathrm{K}$, we used CLUMPP to determine the mean membership probabilities $(Q)$ of each individual at each value of K. Samples from fields $\mathrm{NC}-\mathrm{IVH}, \mathrm{NC}-\mathrm{CH}, \mathrm{MA}$, and ME were consistently placed into separate populations at $\mathrm{K}=6$ to 12. Samples from fields NC-IVH and NC-CH were placed in separate populations at $Q>0.90$ and samples from MA and ME at $Q>0.80$. There was support that samples from several other fields belonged to separate populations: samples from field NY at $Q>0.75$ for $\mathrm{K}=6$ to 12 , NC-WJ at $Q>0.70$ for $\mathrm{K}=9$ to 12 , and NC-IVT at $Q>0.80$ for $\mathrm{K}=8$ to 12. Samples from fields NJ and WA were grouped together at $Q>$ 0.70 for $\mathrm{K}=10$ to 12 , with samples having a $Q>0.70$ at $\mathrm{K}=8$ to 12 for WA and $\mathrm{K}=10$ to 12 for NJ. Samples from MI could not be assigned to a population at $Q>0.70$, but were placed in a separate population at $Q>0.60$ at $\mathrm{K}=11$ and 12 . Overall, results from STRUCTURE support $\mathrm{K}=8$ or 9 , with samples from fields NCIVH, NC-CH, NC-IVT, MA, NY, NC-WJ, and ME and from both $\mathrm{NJ}$ and WA belonging to separate populations, and limited support for samples from MI belonging to another population.

Discriminate analysis of principal components supported $\mathrm{K}=$ 10 clusters based on analyzing samples from the 18 fields and plotting the mean BIC values against K (Fig. 3) Clustering was visualized by producing scatterplots of discriminate functions and a histogram of membership probabilities of the samples to the 10 clusters (Fig. 4). Herein, we refer to clusters 1 to 10 based on their numbering in Figure 4. Samples collected from field $\mathrm{ME}$ and seven fields in the Southeast United States (MS, GA, NC-CW, NC-FR, NC-SM, NC-GR, and NC-WL) were assigned to cluster 6 and were genetically distant from all other clusters. The samples from field NC-WJ were assigned to cluster 8 and also clustered distantly from samples from all other fields. The other eight clusters were more closely grouped, indicating that they were more genetically related to each other than to clusters 6 and 8 . Two clusters were detected within field NC-IVH, with all but two samples from NC-IVH being placed into either cluster 1 or 2 . The majority of samples from a field were assigned to the same cluster: all but two samples from MA were placed in cluster 3, all but one sample in NY in cluster 4, all samples from MI in cluster

TABLE 2. Pairwise $\Phi_{\mathrm{PT}}$ values as calculated with analysis of molecular variance (below diagonal) and geographic distance in kilometers (above diagonal) ${ }^{\mathrm{a}}$

\begin{tabular}{|c|c|c|c|c|c|c|c|c|c|c|}
\hline Field ID & $\mathrm{NC}-\mathrm{CH}$ & NC-IVT & MA & MI & NJ & NY & WA & NC-WJ & $\mathrm{ME}$ & NC-IVH \\
\hline $\mathrm{NC}-\mathrm{CH}$ & - & 44.417 & $1,024.0$ & $1,190.1$ & 664.0 & 989.3 & $3,965.4$ & 372.3 & $1,423.1$ & 54.2 \\
\hline NC-IVT & 0.353 & - & $1,020.0$ & $1,147.8$ & 656.0 & 978.0 & $3,920.9$ & 328.0 & $1,421.9$ & 11.0 \\
\hline MA & 0.300 & 0.238 & - & $1,211.9$ & 369.9 & 170.2 & $3,978.3$ & $1,068.2$ & 407.5 & $1,025.1$ \\
\hline MI & 0.515 & 0.474 & 0.335 & - & $1,033.6$ & $1,044.5$ & $2,855.5$ & 851.2 & $1,505.5$ & $1,140.6$ \\
\hline NJ & 0.359 & 0.381 & 0.167 & 0.449 & - & 329.3 & $3,879.1$ & 706.5 & 777.2 & 660.3 \\
\hline NY & 0.372 & 0.410 & 0.281 & 0.464 & 0.330 & - & $3,810.4$ & 975.5 & 54.7 & 981.4 \\
\hline WA & 0.427 & 0.483 & 0.276 & 0.520 & 0.248 & 0.426 & - & $3,593.4$ & $4,139.1$ & $3,911.4$ \\
\hline NC-WJ & 0.618 & 0.658 & 0.541 & 0.713 & 0.647 & 0.660 & 0.730 & - & $1,473.8$ & 318.2 \\
\hline $\mathrm{ME}$ & 0.535 & 0.546 & 0.442 & 0.628 & 0.545 & 0.533 & 0.610 & 0.613 & - & $1,427.6$ \\
\hline NC-IVH & 0.284 & 0.293 & 0.222 & 0.400 & 0.324 & 0.327 & 0.388 & 0.554 & 0.486 & - \\
\hline
\end{tabular}

a Results are based on a clone-corrected data set using the 10 fields containing $\geq 12$ MLHs. All $\Phi_{\mathrm{PT}}$ values were significant at $P<0.0001$. 
5, all from NC-IVT in cluster 7, and all but one sample from NC$\mathrm{CH}$ in cluster 9. Cluster 10 was comprised of all but four samples from NJ and all samples from OR and WA. These results are similar to those from STRUCTURE with the exceptions that MI was considered a separate population and two populations were detected within field NC-IVH with DAPC. Population differentiation among the 10 clusters was further supported by AMOVA as all pairwise cluster comparisons were highly significant at $P<$ 0.0001 , with $\Phi_{\mathrm{PT}}$ values ranging from 0.177 to 0.679 .

\section{DISCUSSION}

While sexual reproduction is required for $M$. vacciniicorymbosi to complete its life cycle, little information was previously known about the occurrence of sexual recombination within populations of $M$. vaccinii-corymbosi. The presence of numerous MLHs and high haploid diversity within 10 of the sampled fields (NC-IVH, NC-CH, NC-IVT, NC-WJ, MA, ME, MI, NJ, NY, and WA) suggested the presence of sexual recombination within these fields. Random mating was supported within six of the fields (NC-IVT, NC-WJ, MA, MI, ME, and NJ) by linkage equilibrium of microsatellite loci. The possibility of inbreeding and/or self-fertility were suggested in fields $\mathrm{NC}-\mathrm{CH}$, $\mathrm{NC}-\mathrm{IVH}, \mathrm{NY}$, and WA based on the presence of at least one pair of microsatellite markers in linkage disequilibrium when analyzing a non-clone-corrected data set. The presence of several samples with identical MLHs within several fields also provided evidence for self-fertility and/or inbreeding. In particular, 14 loci pairs were in linkage disequilibrium in field NY, and from the 28 samples analyzed from this field only 13 unique MLHs were detected. Linkage disequilibrium was likely not due to physical
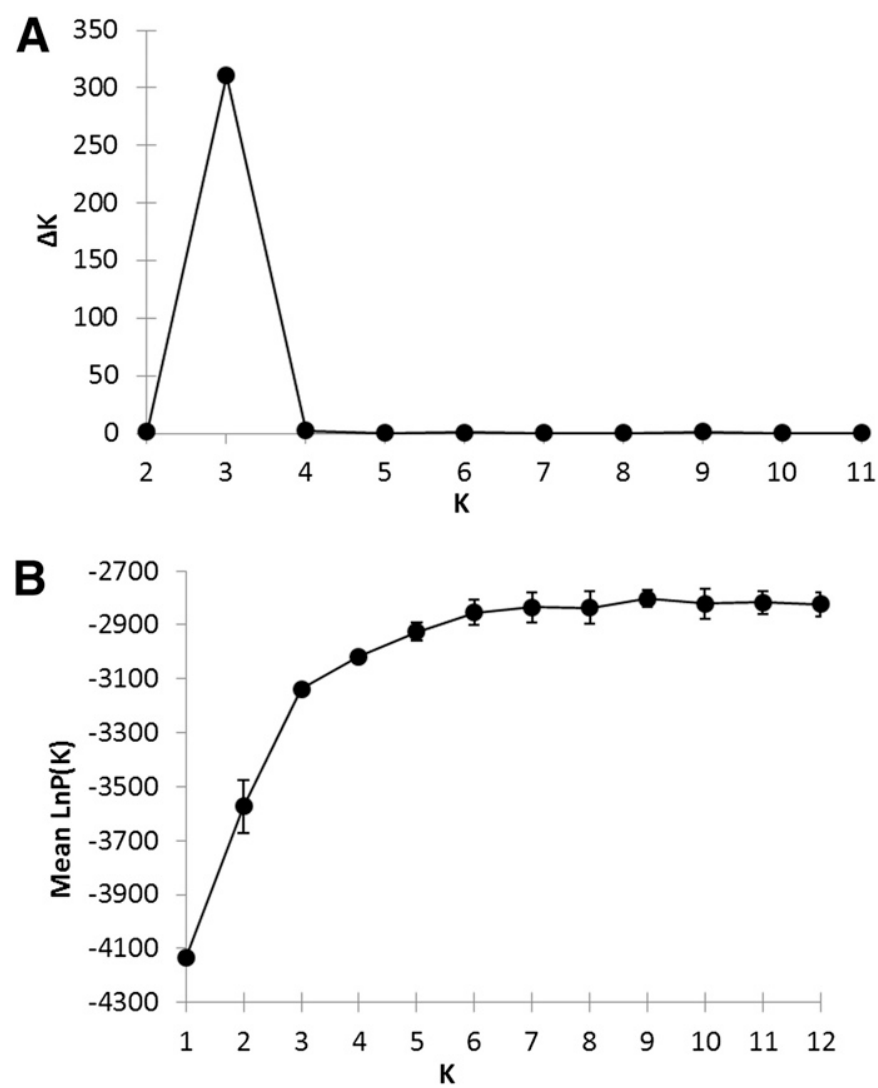

Fig. 1. Results from analysis with the program STRUCTURE. A, Evanno method results based on calculating $\Delta \mathrm{K}$ and plotting against $\mathrm{K}$. B, Mean $\mathrm{LnP}(\mathrm{K})$ plotted against $\mathrm{K}$ for 20 runs at each value of $\mathrm{K}$. Error bars indicate standard deviation of the mean. linkage of the loci during recombination since marker pairs were not consistently in linkage disequilibrium across several fields.

In contrast, very low to no haploid diversity and the presence of only three unique MLHs from 141 samples analyzed from seven fields in the southeastern US (GA, MS, NC-CW, NC-FR, NC-SM, NC-GR, and NC-WL) and only 4 MLHs of 29 analyzed samples from OR suggested inbreeding and/or self-fertility within these fields. Samples from these seven fields in the southeastern United States were genetically very similar or identical to each other, suggesting a large population throughout the sampled geographic region. Several mating systems have been described in fungi, with

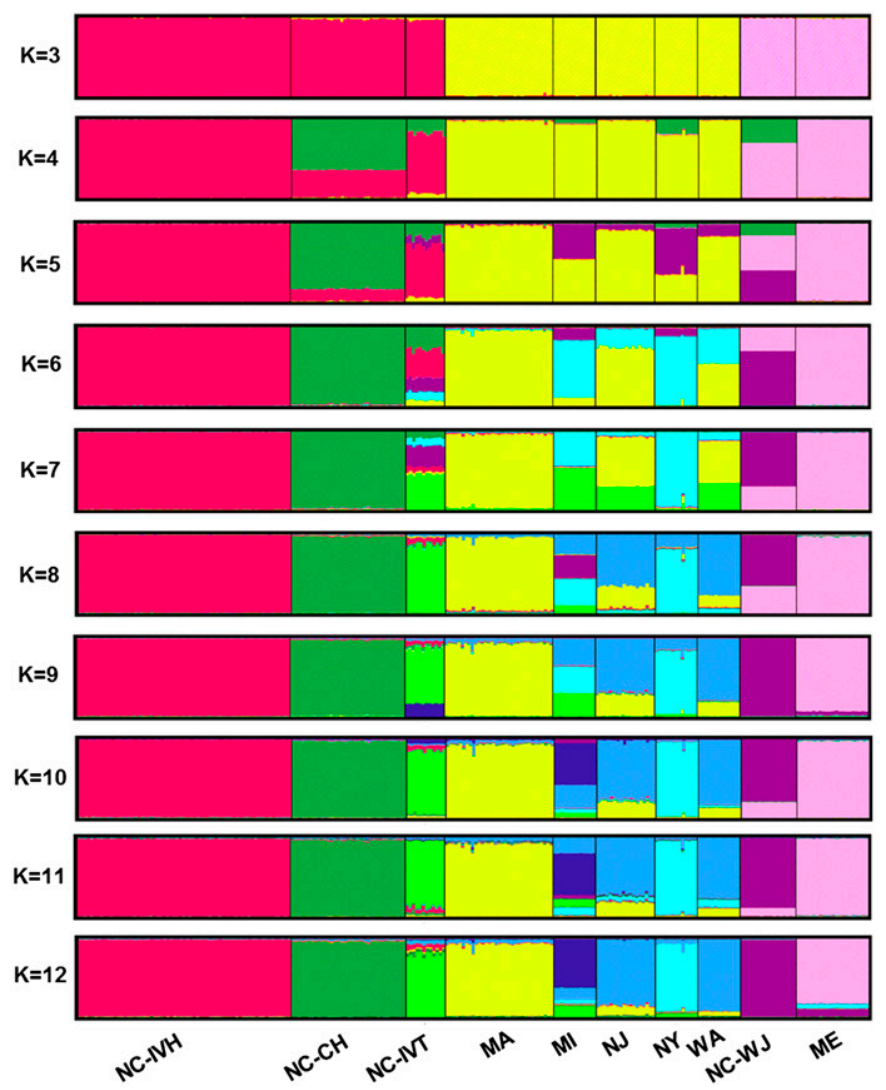

Fig. 2. Membership probability histograms from analysis with STRUCTURE at each value of $\mathrm{K}$ from 3 to 12 . Field abbreviations are based on Table 1.

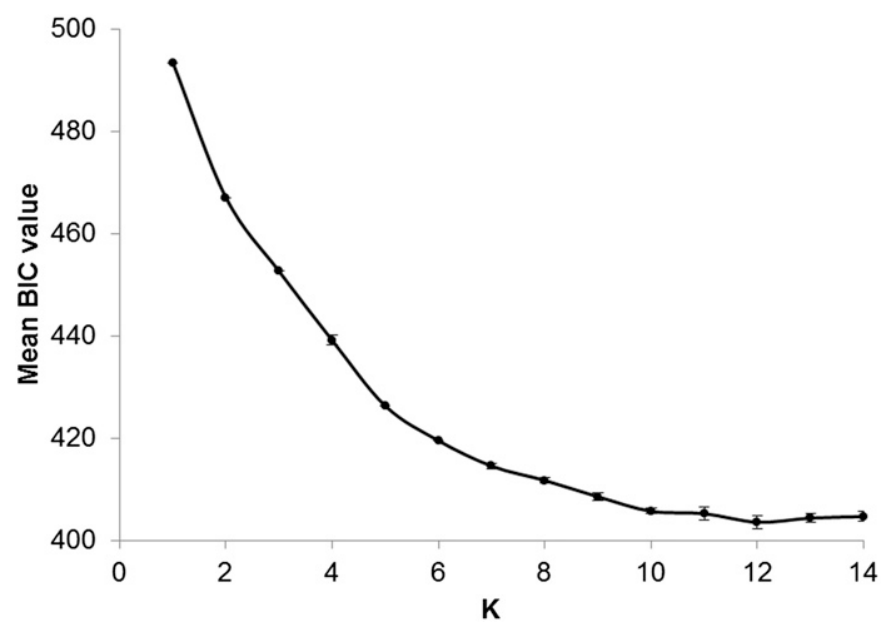

Fig. 3. Mean Bayesian information criterion (BIC) plotted against $\mathrm{K}$ as calculated using the K-means clustering algorithm implemented in adegenet. Error bars indicate standard deviation of the mean. 
homothallism (i.e., the ability to self-fertilize) and heterothallism (i.e., obligate outcrossing with a sexually compatible individual) being the predominant mating systems $(7,23)$. While the mating system of $M$. vaccinii-corymbosi remains unknown, evidence for self-fertility suggests homothallic mating is occurring within the seven fields. However, this does not preclude the presence of heterothallic or another mating system in other populations of M. vaccinii-corymbosi. The closely related genus Sclerotinia contains species that are homothallic, heterothallic, or display a mixed mating system $(1,9,16,25)$. Another closely related fungus, Botrytis cinerea, has a heterothallic mating system based on genome sequencing $(1,25)$. Within the genus Monilinia, outcrossing and heterothallic mating were supported in M. fructicola based on the examination of single ascospore progeny from apothecia (20). High genetic diversity and evidence for sexual recombination and linkage equilibrium in several fields suggests that heterothallic mating may exist in populations of $M$. vacciniicorymbosi. Furthermore, more than one MLH may be present within a single infected fruit and an apothecium can produce sexually recombinant ascospores (K. M. Burchhardt, unpublished data). Overall, our results suggest that populations of $M$. vacciniicorymbosi may differ in their reproductive biology, but further research is needed to determine the mating system within populations of $M$. vaccinii-corymbosi.

Variability in the reproductive biology and population structure of $M$. vaccinii-corymbosi was detected based on geographic region and possibly host species. Samples collected from seven of the fields in the southeastern United States were genetically identical or very similar to each other but were highly genetically differentiated from those from other fields except those from ME. The clustering of samples from these seven southeastern fields with those from ME based on DAPC was surprising considering the geographic distance between the fields and differences in their host. Samples from ME were collected from wild lowbush blueberry ( $V$. angustifolium), while samples from the seven southeastern fields were collected from rabbiteye blueberry ( $V$. virgatum). The range of these two host species only overlaps in North Carolina (USDA, NRCS), and analysis of genetic relatedness of cultivated blueberries supported that cultivars of $V$. virgatum are genetically divergent from $V$. angustifolium as well as northern highbush blueberry (V. corymbosum) (6). However, it is important to note that while the samples from ME genetically clustered with those from seven fields in the Southeast their reproductive biology was different, with high genetic diversity and linkage equilibrium within ME supporting sexual recombination and low genetic diversity in the seven Southeast fields supporting self-fertilization. There are several plausible explanations for the grouping of samples from ME with those from the seven sites in the Southeast. It is possible that $M$. vaccinii-corymbosi underwent a geographic and host range expansion that may have been coupled with a shift in its reproductive biology. Another possibility is that M. vaccinii-corymbosi was recently introduced from $V$. angustifolium onto $V$. virgatum in the southeastern United States and that low genetic diversity is due to a founder effect. However, this explanation seems unlikely considering the geographic expansiveness of the population in the Southeast. Finally, another explanation is that DAPC was not able to detect underlying population structuring between the seven southeastern fields and ME. The presence of private alleles in a high frequency within both ME and the Southeast population supports this hypothesis, as the frequency of private alleles may be an indicator of level of gene flow (49).

Low genetic diversity and strong genetic similarity of $M$. vaccinii-corymbosi isolates from seven fields in the southeastern United States and genetic divergence of these isolates from northern $M$. vaccinii-corymbosi populations may be explained by several scenarios. One scenario is that a founder effect is responsible for low genetic diversity due to a recent introduction and spread of $M$. vaccinii-corymbosi throughout the Southeast. This scenario is unlikely based on the large geographic extent of the Southeast population. Considering the strong genetic divergence of the Southeast population from other $M$. vaccinii-corymbosi populations, another possibility is that cryptic species exist
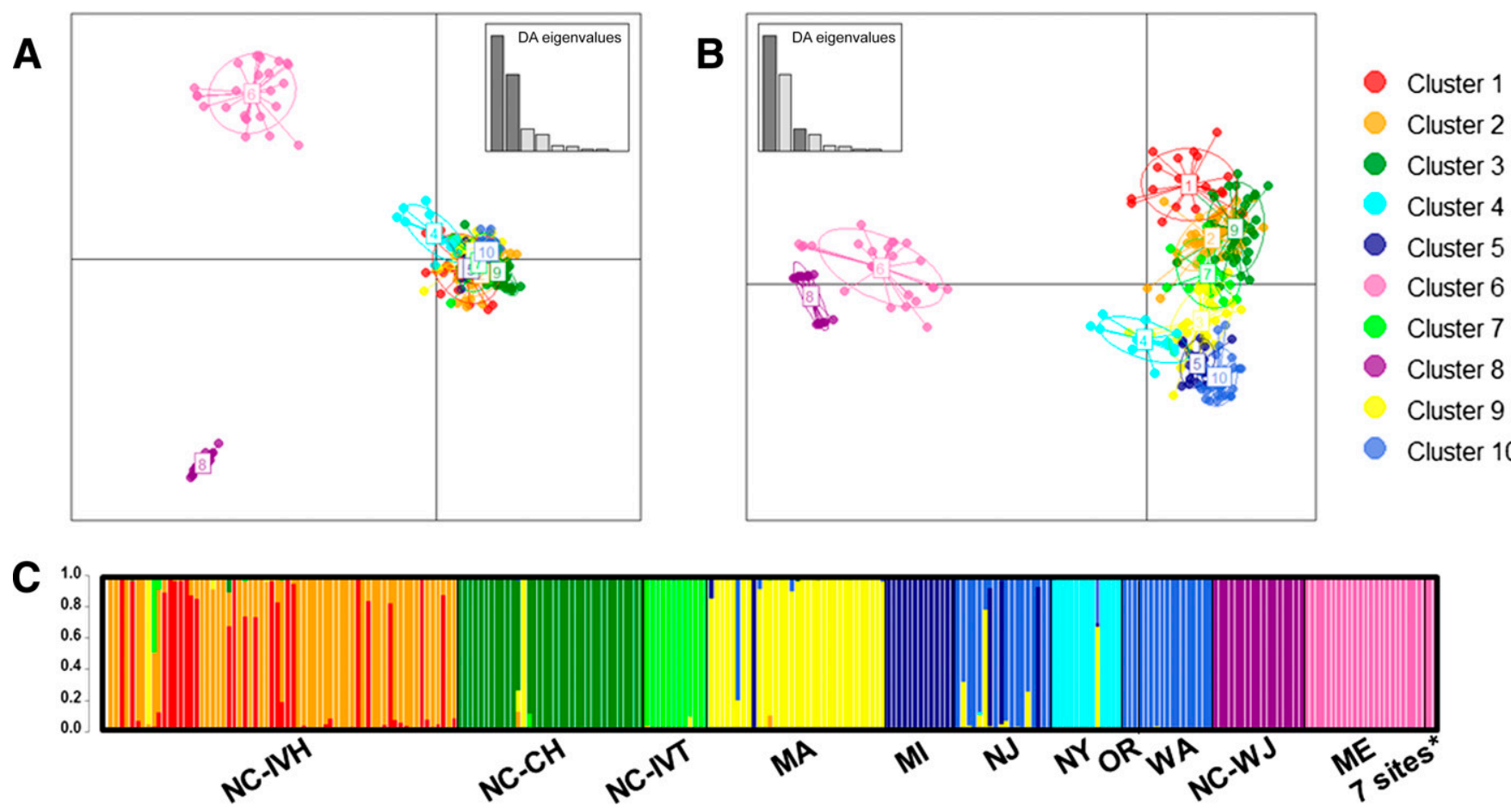

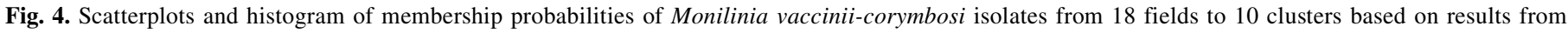

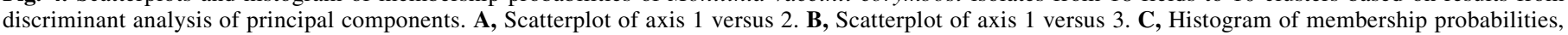

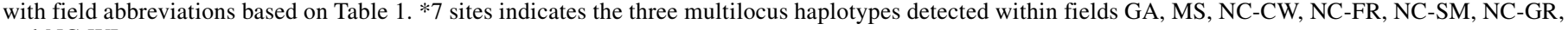
and NC-WL. 
within $M$. vaccinii-corymbosi that are reproductively isolated, thus preventing genetic exchange. Mating experiments involving sexual crosses are needed along with additional genetic analyses to test for reproductive isolation of genetically divergent isolates of $M$. vaccinii-corymbosi. It is also possible that host specificity is driving genetic divergence and/or cryptic speciation. Cryptic speciation was detected in the fungus Botrytis cinerea based on genetic differentiation of populations on Vitis vinifera and Rubus fructicosus grown in sympatry and inability to mate $(19,55)$. Interestingly, all isolates of M. vaccinii-corymbosi from seven fields in the Southeast with low genetic diversity were collected from infected shoots or fruit of V. virgatum. Further support for this scenario is that $M$. vaccinii-corymbosi samples collected from $V$. virgatum fruit in fields NC-GR and NC-WL were located within approximately $25 \mathrm{~km}$ of fields NC-IVH and NC-IVT, where samples were collected from shoots of a V. corymbosum $\times$ $V$. darrowii hybrid and shoots of $V$. virgatum, respectively. A single MLH was detected within fields NC-GR and NC-WL that was also detected in fields MS, GA, and NC-CW and was genetically divergent from $M$. vaccinii-corymbosi samples from fields NC-IVH and NC-IVT, which contained high genetic diversity and haplotypes that were more genetically similar to $M$. vaccinii-corymbosi isolates from northern fields. It is possible that the $M$. vaccinii-corymbosi isolates on $V$. virgatum in the Southeast specifically infect the shoots and fruit of $V$. virgatum, while $M$. vaccinii-corymbosi isolates on $V$. corymbosum and $V$. corymbosum $\times V$. darrowii can infect the shoots and fruit of their host of origin as well as the shoots of $V$. virgatum, but not the fruit of $V$. virgatum. Research suggests that cultivars of $V$. virgatum tend to be susceptible to shoot infection (14), with a history of severe shoot infection in southeastern North Carolina (33). Little to no fruit infection was reported on V. virgatum in Southeast North Carolina until 2012 when we sampled from fields NC-GR and NC-WL (K. M. Burchhardt, B. Cline, and M. A. Cubeta, unpublished data). However, genetic divergence of $M$. vaccinii-corymbosi isolates originating from different hosts grown in close proximity can be due to other factors than reproductive barriers, such as temporal isolation. Variation in timing of bud break and flower bloom between $V$. corymbosum and $V$. virgatum and timing of apothecia production from pseudosclerotia produced on hosts with different developmental phenologies is known to exist $(30,31,46,53)$. If asexual and/or sexual spores of $M$. vaccinii-corymbosi are produced at a time when one plant host species is susceptible while another is not, this could potentially drive temporal isolation and genetic divergence of M. vaccinii-corymbosi populations. In the insect vectored anther smut fungus Microbotryum violaceum, genetic differentiation of populations on the plant hosts Silene dioica and S. latifolia grown in sympatry was not due to reproductive isolation, but rather other factors such as host phenology and insect vector behavior $(21,54)$. Inoculation experiments would be necessary to test the hypothesis of host specificity of isolates of $M$. vaccinii-corymbosi during fruit infection and also to determine if reproductive isolation and cryptic speciation exists within $M$. vaccinii-corymbosi.

The lack of isolation by distance found in this study suggests that geographic distance between fields does not necessarily indicate the level of gene flow among fields. Since research suggests that $M$. vaccinii-corymbosi cannot survive in its host systemically (2), methods of gene flow could be through aerial dispersal of ascospores, movement of conidia by insects and other potential vectors, transportation of infected plants, or movement of pseudosclerotia. The $V$. corymbosum breeding program was started in approximately 1908 when plants were selected from the pine barrens in New Jersey for development of cultivated varieties (53). The natural range of $V$. corymbosum covers eastern North America, with cultivars of $V$. corymbosum introduced into the Pacific Northwest (USDA GRIN). Interestingly, DAPC and STRUCTURE supported that isolates of $M$. vaccinii-corymbosi collected from fields in OR and WA clustered with those from NJ. Lower levels of genetic diversity in OR compared with WA may be the result of a founder effect due to a recent introduction of $M$. vaccinii-corymbosi into OR. However, further sampling and genetic analyses are needed to examine migration rates into and throughout the Pacific Northwest.

Multiple analytical approaches were applied to examine the presence of population structure of $M$. vaccinii-corymbosi. Multivariate analysis with DAPC supported further population structure than Bayesian analysis with STRUCTURE. Research suggests that DAPC is more effective than STRUCTURE at detecting hierarchical structuring (28). Another potential benefit of DAPC is that it does not have evolutionary assumptions such as random mating built into the analysis as with STRUCTURE (28). Complex hierarchical structuring and possible violations in the assumptions of the model implemented with STRUCTURE may have caused variation in results from multiple runs at a given value of $\mathrm{K}$. While the Evanno method is commonly used in population genetic publications to determine $\mathrm{K}$ from STRUCTURE output, our data shows the importance of considering numerous methods and analytical approaches to determine $\mathrm{K}$. The Evanno method supported $\mathrm{K}=3$ and grouped NC-WJ and ME, two fields that were genetically distinct based on DAPC and AMOVA. In our study, further population structuring was supported by considering the $\ln \mathrm{P}(\mathrm{K})$ and membership probabilities of the samples to populations. Using this approach, results from DAPC and STRUCTURE were similar, with $\mathrm{K}=10$ supported with DAPC and $\mathrm{K}=8$ or 9 supported for STRUCTURE. A difference between results obtained from DAPC and STRUCTURE was that DAPC placed isolates of $M$. vaccinii-corymbosi from field MI in a distinct cluster while population assignment of MI was inconclusive with STRUCTURE. Another difference was that two genetically distinct clusters were detected within field NC-IVH with DAPC. Multiple introductions of $M$. vaccinii-corymbosi into field NC-IVH may explain this observation.

A previous study by Wasilwa et al. (56) used amplified fragment length polymorphism markers and vegetative compatibility tests to examine genetic structuring of 191 isolates of M. vacciniicorymbosi from several locations in North America. They concluded that intraspecific genetic diversity of $M$. vaccinii-corymbosi is limited in North America. In contrast, our study found that polymorphic microsatellite markers were able to detect genetic diversity and structuring of populations of M. vaccinii-corymbosi. The possibility of host specificity needs to be examined further and is of practical significance for plant breeders interested in developing blueberry cultivars with resistance to $M$. vaccinii-corymbosi. Current breeding programs focus primarily on screening cultivars of Vaccinium species for their level of susceptibility to shoot and fruit infection $(12-15,50,51)$. However, the majority of these studies have been conducted in a single location using local sources of inoculum. For results to be reliable in several geographic regions and on different host species it is important to screen plants with isolates of $M$. vaccinii-corymbosi that broadly represent the phenotypic and genotypic diversity of pathogen populations $(8,32,57)$. The extent to which genetic divergence of $M$. vaccinii-corymbosi populations reflects phenotypic variability in terms of aggressiveness, developmental and reproductive biology of $M$. vaccinii-corymbosi remains to be determined. The results from this study provide a foundation of knowledge for future studies on the disease ecology and population biology of M. vaccinii-corymbosi.

\section{ACKNOWLEDGMENTS}

We thank B. Cline, M. McCormick, A. Putman, E. Thomas, P. Ojiambo, I. Carbone, N. Singh, and K. Koelle for their advice on this project; and L. McDermott, A. Durden, M. Butler, G. Moulton, B. Bloodworth, H. Scherm, S. Annis, N. Grunwald, A. Schilder, P. Oudemans, J. Polashock, and B. Smith, as well as several blueberry growers for their help collecting samples. Funding for this project was 
provided by an NCDA\&CS Specialty Crops Block Grant to M. A. Cubeta. K. M. Burchhardt was financially supported by a USDA National Needs Fellowship and PEO Scholar Award during her graduate studies.

\section{LITERATURE CITED}

1. Amselem, J., Cuomo, C. A., van Kan, J. A. L., Viaud, M., Benito, E. P., Couloux, A., Coutinho, P. M., de Vries, R. P., Dyer, P. S., Fillinger, S., Fournier, E., Gout, L., Hahn, M., Kohn, L., Lapalu, N., Plummer, K. M., Pradier, J.-M., Quévillon, E., Sharon, A., Simon, A., ten Have, A., Tudzynski, B., Tudzynski, P., Wincker, P., Andrew, M., Anthouard, V., Beever, R. E., Beffa, R., Benoit, I., Bouzid, O., Brault, B., Chen, Z., Choquer, M., Collémare, J., Cotton, P., Danchin, E. G., Da Silva, C., Gautier, A., Giraud, C., Giraud, T., Gonzalez, C., Grossetete, S., Güldener, U., Henrissat, B., Howlett, B. J., Kodira, C., Kretschmer, M., Lappartient, A., Leroch, M., Levis, C., Mauceli, E., Neuvéglise, C., Oeser, B., Pearson, M., Poulain, J., Poussereau, N., Quesneville, H., Rascle, C., Schumacher, J., Ségurens, B., Sexton, A., Silva, E., Sirven, C., Soanes, D. M., Talbot, N. J., Templeton, M., Yandava, C., Yarden, O., Zeng, Q., Rollins, J. A., Lebrun, M.-H., and Dickman, M. 2011. Genomic analysis of the necrotrophic fungal pathogens Sclerotinia sclerotiorum and Botrytis cinerea. PLoS Genet. 7:e1002230.

2. Batra, L. R. 1983. Monilinia vaccinii-corymbosi - Its biology on blueberry and comparison with related species. Mycologia 75:131-152.

3. Batra, L. R. 1991. World species of Monilinia (Fungi): their ecology, biosystematics and control. Vol. Mycologia Memoir No. 16, Mycologia Memoir No. 16. J. Cramer, Berlin.

4. Batra, L. R., and Batra, S. W. T. 1985. Floral mimicry induced by mummyberry fungus exploits hosts pollinators as vectors. Science 228:1011-1013.

5. Benjamini, Y., and Hochberg, Y. 1995. Controlling the false discovery rate- a practical and powerful approach to multiple testing. J. R. Stat. Soc., B 57:289-300.

6. Bian, Y. 2012. Genetic diversity and population structure of cultivated blueberries (Vaccinium section Cyanococcus spp.), Horticultural Science. North Carolina State University, North Carolina.

7. Billiard, S., Lopez-Villavicencio, M., Hood, M. E., and Giraud, T. 2012. Sex, outcrossing and mating types: unsolved questions in fungi and beyond. J. Evol. Biol. 25:1020-1038.

8. Chakraborty, S., Liu, C. J., Mitter, V., Scott, J. B., Akinsanmi, O. A., Ali, S., Dill-Macky, R., Nicol, J., Backhouse, D., and Simpfendorfer, S. 2006. Pathogen population structure and epidemiology are keys to wheat crown rot and Fusarium head blight management. Australas. Plant Pathol. 35:643-655.

9. Chitrampalam, P., Inderbitzin, P., Maruthachalam, K., Wu, B.-M., and Subbarao, K. V. 2013. The Sclerotinia sclerotiorum mating type locus $(M A T)$ contains a 3.6-kb region that is inverted in every meiotic generation. PLoS ONE 8:e56895.

10. Cox, K. D., and Scherm, H. 2001. Gradients of primary and secondary infection by Monilinia vaccinii-corymbosi from point sources of ascospores and conidia. Plant Dis. 85:955-959.

11. Earl, D. A., and Vonholdt, B. M. 2012. STRUCTURE HARVESTER: A website and program for visualizing STRUCTURE output and implementing the Evanno method. Conserv. Genet. Resour. 4:359-361.

12. Ehlenfeldt, M. K., Polashock, J. J., Stretch, A. W., and Kramer, M. 2010. Mummy berry fruit rot and shoot blight incidence in blueberry: prediction, ranking, and stability in a long-term study. HortScience 45: 92-97.

13. Ehlenfeldt, M. K., Polashock, J. J., Stretch, A. W., and Kramer, M. 2010. Ranking cultivated blueberry for mummy berry blight and fruit infection incidence using resampling and principal components analysis. HortScience 45:1205-1210.

14. Ehlenfeldt, M. K., and Stretch, A. W. 2000. Mummy berry blight resistance in rabbiteye blueberry cultivars. HortScience 35:1326-1328.

15. Ehlenfeldt, M. K., and Stretch, A. W. 2001. Resistance to blighting by Monilinia vaccinii-corymbosi in diploid and polyploid Vaccinium species. HortScience 36:955-957.

16. Ekins, M., Aitken, E. A., and Coulter, K. C. 2006. Homothallism in Sclerotinia minor. Mycol. Res. 110:1193-1199.

17. Evanno, G., Regnaut, S., and Goudet, J. 2005. Detecting the number of clusters of individuals using the software STRUCTURE: a simulation study. Mol. Ecol. 14:2611-2620.

18. Faircloth, B. C. 2008. MSATCOMMANDER: Detection of microsatellite repeat arrays and automated, locus-specific primer design. Mol. Ecol. Resour. 8:92-94.

19. Fournier, E., and Giraud, T. 2008. Sympatric genetic differentiation of a generalist pathogenic fungus, Botrytis cinerea, on two different host plants, grapevine and bramble. J. Evol. Biol. 21:122-132.

20. Free, S. J., Holtz, B. A., and Michailides, T. J. 1996. Mating behavior in field populations of Monilinia fructicola. Mycologia 88:208-211.
21. Gladieux, P., Vercken, E., Fontaine, M. C., Hood, M. E., Jonot, O., Couloux, A., and Giraud, T. 2011. Maintenance of fungal pathogen species that are specialized to different hosts: allopatric divergence and introgression through secondary contact. Mol. Biol. Evol. 28: 459-471.

22. Gosch, C. 2003. Monilinia vaccinii-corymbosi on Highbush blueberries (Vaccinium corymbosum L.): Also in Europe. Eur. J. Hortic. Sci. 68: 238-241.

23. Heitman, J., Kronstad, J. W., Taylor, J. W., and Casselton, L. A. 2007. Sex in fungi: molecular determination and evolutionary implications. ASM Press, Washington.

24. Hildebrand, P. D., and Braun, P. G. 1991. Factors affecting infection of lowbush blueberry by ascospores of Monilinia vaccinii-corymbosi. Can. J. Plant Pathol. 13:232-240.

25. Holst-Jensen, A., Kohn, L. M., Jakobsen, K. S., and Schumacher, T. 1997. Molecular phylogeny and evolution of Monilinia (Sclerotiniaceae) based on coding and noncoding rDNA sequences. Am. J. Bot. 84: 686-701.

26. Jakobsson, M., and Rosenberg, N. A. 2007. CLUMPP: a cluster matching and permutation program for dealing with label switching and multimodality in analysis of population structure. Bioinformatics 23:18011806.

27. Jombart, T. 2008. adegenet: An R package for the multivariate analysis of genetic markers. Bioinformatics 24:1403-1405.

28. Jombart, T., Devillard, S., and Balloux, F. 2010. Discriminant analysis of principal components: a new method for the analysis of genetically structured populations. BMC Genet. 11.

29. Kretzer, A. M., Molina, R., and Spatafora, J. W. 2000. Microsatellite markers for the ectomycorrhizal basidiomycete Rhizopogon vinicolor. Mol. Ecol. 9:1190-1191.

30. Lehman, J. S., and Oudemans, P. V. 1997. Phenology of apothecium production in populations of Monilinia vaccinii-corymbosi from earlyand late-maturing blueberry cultivars. Phytopathology 87:218-223.

31. Lehman, J. S., and Oudemans, P. V. 2000. Variation and heritability of phenology in the fungus Monilinia vaccinii-corymbosi on blueberry. Phytopathology 90:390-395.

32. Milgroom, M. G., and Peever, T. L. 2003. Population biology of plant pathogens - The synthesis of plant disease epidemiology and population genetics. Plant Dis. 87:608-617.

33. Milholland, R. D. 1977. Sclerotium germination and histopathology of Monilinia-vaccinii-corymbosi on highbush blueberry. Phytopathology 67: 848-854.

34. Molecular Ecology Resources Primer Development Consortium, Abelló, P., Ai, W., Altmann, C., Bernardi, G., Bonato, O., Burchhardt, K. M., Chen, X., Chen, Z., Čížková, D., Clouet, C., Cubeta, M. A., GarciaMerchan, V. H., Gauthier, N., Gibson, S., Halačka, K., Hamdi, F., Hankeln, T., Hochkirch, A., Hrbek, T., Jackson, A. M., Lin, C., Lin, S.-M., Macpherson, E., Macrander, J., Marešová, E. V. A., Mendel, J. A. N., Nowak, M., Orti, G., Palero, F., Papoušek, I. V. O., Pascual, M., Schmitt, T., Semmens, B. X., Streito, J.-C., Tian, E.-W., Tseng, S.-P., Veith, M., Vetešník, L., Wang, H.-Y., Weyer, J., Willis, S., Yu, H. U. I., and Zhou, Z. 2012. Permanent Genetic Resources added to Molecular Ecology Resources Database 1 April 2012 - 31 May 2012. Mol. Ecol. Resour. 12: 972-974.

35. Ngugi, H. K., and Scherm, H. 2004. Pollen mimicry during infection of blueberry flowers by conidia of Monilinia vaccinii-corymbosi. Physiol. Mol. Plant Pathol. 64:113-123.

36. Ngugi, H. K., and Scherm, H. 2006. Mimicry in plant-parasitic fungi. FEMS Microbiol. Lett. 257:171-176.

37. Ngugi, H. K., Scherm, H., and Lehman, J. S. 2002. Relationships between blueberry flower age, pollination, and conidial infection by Monilinia vaccinii-corymbosi. Phytopathology 92:1104-1109.

38. Peakall, R., and Smouse, P. E. 2006. GENALEX 6: Genetic analysis in Excel. Population genetic software for teaching and research. Mol. Ecol. Notes 6:288-295.

39. Peakall, R., and Smouse, P. E. 2012. GenAlEx 6.5: genetic analysis in Excel. Population genetic software for teaching and research- an update. Bioinformatics 28:2537-2539.

40. Penman, L. N., and Annis, S. L. 2005. Leaf and flower blight caused by Monilinia vaccinii-corymbosi on lowbush blueberry: Effects on yield and relationship to bud phenology. Phytopathology 95:1174-1182.

41. Pritchard, J. K., Stephens, M., and Donnelly, P. 2000. Inference of population structure using multilocus genotype data. Genetics 155: 945-959.

42. Raymond, M., and Rousset, F. 1995. GENEPOP (version 1.2): population genetics software for exact tests and ecumenicism. J. Hered. 86: 248-249.

43. Rosenberg, N. A. 2003. DISTRUCT: A program for the graphical display of population structure. Mol. Ecol. Notes 4:137-138. 
44. Rosenberg, N. A., Pritchard, J. K., Weber, J. L., Cann, H. M., Kidd, K. K., Zhivotovsky, L. A., and Feldman, M. W. 2002. Genetic structure of human populations. Science 298:2381-2385.

45. Rousset, F. 2008. Genepop'007: a complete reimplementation of the Genepop software for Windows and Linux. Mol. Ecol. Resour. 8:103-106.

46. Scherm, H., Savelle, A. T., and Pusey, P. L. 2001. Interactions between chill-hours and degree-days affect carpogenic germination in Monilinia vaccinii-corymbosi. Phytopathology 91:77-83.

47. Schuelke, M. 2000. An economic method for the fluorescent labeling of PCR fragments. Nat. Biotechnol. 18:233-234.

48. Shinners, T. C., and Olson, A. R. 1996. The gynoecial infection pathway of Monilinia vaccinii-corymbosi in lowbush blueberry (Vaccinium angustifolium). Can. J. Plant Sci. 76:493-497.

49. Slatkin, M. 1985. Rare alleles as indicators of gene flow. Evolution 39:53-65.

50. Stretch, A. W., and Ehlenfeldt, M. K. 2000. Resistance to the fruit infection phase of mummy berry disease in highbush blueberry cultivars. HortScience 35:1271-1273.

51. Stretch, A. W., Ehlenfeldt, M. K., Brewster, V., Vorsa, N., and Polashock, J. 2001. Resistance of diploid Vaccinium spp. to the fruit rot stage of mummy berry disease. Plant Dis. 85:27-30.
52. Strik, B. C. and Yarborough, D. 2005. Blueberry production trends in North America, 1992 to 2003, and predictions for growth. Horttechnology 15:391-398.

53. Trehane, J. 2004. Blueberries, Cranberries, and Other Vacciniums. Royal Horticultural Society Plant Collector Guide. Timber Press, Portland, OR.

54. van Putten, W. F., Elzinga, J. A., and Biere, A. 2007. Host fidelity of the pollinator guilds of Silene dioica and Silene latifolia: Possible consequences for sympatric host race differentiation of a vectored plant disease. Int. J. Plant Sci. 168:421-434.

55. Walker, A. S., Gautier, A., Confais, J., Martinho, D., Viaud, M., Le Pecheur, P., Dupont, J., and Fournier, E. 2011. Botrytis pseudocinerea, a new cryptic species causing gray mold in French vineyards in sympatry with Botrytis cinerea. Phytopathology 101:1433-1445.

56. Wasilwa, L. A., Oudemans, P. V., and Lehman, J. S. 2003. Genetic structure and population diversity of mummy berry (Monilinia) collections from the United States of America and Canada. East Afr. Agric. For. J. 68:125-134.

57. Zhan, J., Fitt, B. D. L., Pinnschmidt, H. O., Oxley, S. J. P., and Newton, A. C. 2008. Resistance, epidemiology and sustainable management of Rhynchosporium secalis populations on barley. Plant Pathol. 57:1-14. 